\title{
Epithelial-Specific TLR4 Knockout Challenges Current Evidence of TLR4 Homeostatic Control of Gut Permeability
}

\author{
Elise E. Crame ${ }^{a}$ Joanne M. Bowen ${ }^{a}$ Kate R. Secombe ${ }^{a}$ Janet K. Coller ${ }^{b}$ \\ Maxime François ${ }^{c, d}$ Wayne Leifert ${ }^{c, d}$ Hannah R. Wardilla, e
}

${ }^{a}$ Discipline of Physiology, School of Biomedicine, The University of Adelaide, Adelaide, SA, Australia; ${ }^{b}$ Discipline of Pharmacology, School of Biomedicine, The University of Adelaide, Adelaide, SA, Australia; 'CSIRO Health \& Biosecurity, Nutrition and Health Program, Molecular Diagnostic Solutions Group, Adelaide, SA, Australia; dDepartment of Molecular and Biomedical Science, School of Biological Science, The University of Adelaide, Adelaide, SA, Australia; 'Department of Paediatric Oncology/Haematology, The University of Groningen, University Medical Centre Groningen, Groningen, The Netherlands

\section{Keywords}

Toll-like receptor 4 - Mucosal barrier function .

Gastrointestinal homeostasis · Ussing chamber · Pre-clinical model

\begin{abstract}
Introduction: Toll-like receptor 4 (TLR4) is a highly conserved immunosurveillance protein of innate immunity, displaying well-established roles in homeostasis and intestinal inflammation. Current evidence shows complex relationships between TLR4 activation, maintenance of health, and disease progression; however, it commonly overlooks the importance of site-specific TLR4 expression. This omission has the potential to influence translation of results as previous evidence shows the differing and distinct roles that TLR4 exhibits are dependent on its spatiotemporal expression. Methods: An intestinal epithelial TLR4 conditional knockout (KO) mouse line ( $\left.T / r 4^{\triangle I E C}, n=6-8\right)$ was utilized to dissect the contribution of epithelial TLR4 expression to intestinal homeostasis with comparisons to wild-type (WT) $(n=5-7)$ counter-
\end{abstract}

karger@karger.com www.karger.com/iid

Karger $\frac{1}{6}$
(C) 2021 The Author(s)

Published by S. Karger AG, Basel

This is an Open Access article licensed under the Creative Commons Attribution-NonCommercial-4.0 International License (CC BY-NC) (http://www.karger.com/Services/OpenAccessLicense), applicable to the online version of the article only. Usage and distribution for commercial purposes requires written permission. parts. Functions of the intestinal barrier in the ileum and colon were assessed with tissue resistance in Ussing chambers. Molecular and structural comparisons in the ileum and colon were assessed via histological staining, expression of tight junction proteins (occludin and zonular occludin 1 [ZO-1]), and presence of CD11b-positive immune cells. Results: There was no impact of the intestinal epithelial TLR4 KO, with no differences in (1) tissue resistance-ileum (mean \pm standard error of mean [SEM]): WT $22 \pm 7.2$ versus TIr $4^{\triangle I E C} 20 \pm 5.6$ $\left(\Omega \times \mathrm{cm}^{2}\right) p=0.831$, colon WT $30.8 \pm 3.6$ versus TIr $4^{\Delta I E C}$ $45.1 \pm 9.5 p=0.191$; (2) histological staining (overall tissue structure); and (3) tight junction protein expression (\% area stain, mean \pm SEM)-ZO-1: ileum-WT $1.49 \pm 0.155$ versus TIr $4^{\triangle I E C} 1.17 \pm 0.07, p=0.09$; colon-WT $1.36 \pm 0.26$ versus TIr $4^{\Delta I E C} 1.12 \pm 0.18 p=0.47$; occludin: ileum-WT $1.07 \pm 0.12$ versus TIr $4^{\Delta I E C} 0.95 \pm 0.13, p=0.53$; colon-WT $1.26 \pm 0.26$ versus TIr4 ${ }^{\triangle I E C} 1.02 \pm 0.16 p=0.45$. CD11b-positive immune cells (\% area stain, mean \pm SEM) in the ileum were mildly decreased in WT mice: WT $0.14 \pm 0.02$ versus TIr $4^{\Delta I E C} 0.09 \pm 0.01$ $p=0.04$. However, in the colon, there was no difference in CD11b-positive immune cells between strains: WT $0.53 \pm 0.08$ 
versus TIr $4^{\triangle I E C} 0.49 \pm 0.08 p=0.73$. Conclusions: These data have 2 important implications. First, these data refute the assumption that epithelial TLR4 exerts physiological control of intestinal physiology and immunity in health. Second, and most importantly, these data support the use of the T/r $4^{\Delta I E C}$ line in future models interrogating health and disease, confirming no confounding effects of genetic manipulation.

(c) 2021 The Author(s)

Published by S. Karger AG, Basel

\section{Introduction}

Polarized epithelial cells covering the intestinal tract form a highly selective barrier between the bacteria-filled gut lumen and the comparatively sterile subepithelial tissue [1]. This barrier maintains homeostasis within the gastrointestinal tract, allowing for nutrient absorption and regulation of water exchange [2]. Crucially, the intestinal epithelial lining is also a first-line of defense from pathogens, whereby innate immune pattern recognition receptors recognize harmful bacteria and promote protective inflammatory cascades [1]. Toll-like receptor 4 (TLR4) is a type of pattern recognition receptor expressed on a variety of cell types including immune [3] and epithelial cells [4]. TLR4 and its accessory proteins MD2 and CD14 are widely researched due to dual roles in homeostatic control and suspected involvement in multiple conditions, including inflammatory bowel diseases and chemotherapy-induced gastrointestinal toxicity $[5,6]$.

Based on its consistent implication with diseased states that are characterized by intestinal dysfunction, TLR4 has been regularly reported to be a key regulator of mucosal barrier function and thus intestinal permeability under physiological conditions $[7,8]$. Intestinal permeability via the paracellular route is dictated via tight junction proteins located on the apical-lateral cell surface [9]. The multiple intercellular and bridging proteins of the tight junction, including occludin, zonular occludin 1 (ZO-1), and claudins, allow for the movement of solutes across their electro-osmotic gradient to maintain intestinal homeostasis [10]. A considerable body of evidence anecdotally supports TLR4-mediated barrier control, with TLR4 expression strongly correlating with functional assessments of intestinal permeability and molecular characteristics of tight junction proteins [6]. For example, a 2018 study by Bein et al. [11] found that in a necrotizing enterocolitis model, a decrease in TLR4 expression was significantly associated with a decrease in occludin, ZO-1, and claudin- 4 and resulted in increased permeability. While this suggests a connection between functional
TLR4 and the preservation of the tight junction complex, these findings are only secondary to original aims and do not fully explain the role of TLR4 in homeostasis. Previous research using global TLR4 knockout $(\mathrm{KO})$ mice also shows that a lack of TLR4 expression does not impact tight junction protein development and barrier function; however, this study only analyzed tight junction expression post-chemotherapy challenges [6]. While these studies implicate TLR4 in the pathobiological control of the mucosal barrier, the majority of these data have been generated in models of disease, and as such, conclusions regarding its physiological control cannot be made.

Another major oversight in the literature regarding TLR4's regulatory control of the intestinal barrier is the lack of site-specific interrogation. TLR4 is not only expressed on epithelial cells of the intestinal mucosa but also immune cells of the submucosa. In fact, immune expression of TLR4 is considerably higher than that of epithelial expression [3], and as such, its impact on mucosal homeostasis and disease is arguably higher. A failure to address site-specific TLR4 mechanisms hampers our ability to dissect causative mechanisms and thus impairs translation of fundamental findings. This has the potential to misguide new interventions targeting TLR4 that may not be delivered in a manner that optimally targets TLR4. This paradox is particularly important in cancer research, where TLR4-dependent mechanisms are central to both the efficacy and toxicity of therapy; yet, a lack of site-specific interrogation has resulted in highly variable and contradictory findings in studies attempting to augment its activity.

There is a clear need to study TLR4-dependent control of the mucosal barrier in a manner that dissects epithelial versus immune mechanisms. As such, we have utilized a conditional intestinal epithelial-specific TLR4 KO mouse line $\left(T l r 4^{\triangle I E C}\right)[12]$, with epithelial deletion of TLR4 and unimpaired immune cell expression TLR4. In characterizing this mouse line, we are given the unique opportunity to rigorously define the regulatory role of epithelial TLR4 on the intestinal barrier under physiological conditions. As such, we aimed to characterize the potential intestinal differences of this $T l r 4^{\Delta I E C}$ line compared to wildtype (WT) mice, using structural, molecular, and electrophysiological assessments.

\section{Materials and Methods}

Animal Husbandry

Male and female WT C57BL/6 $(n=5-7)$ and intestinal epithelial conditional TLR4 KO C57BL/6 (Tlr4 $\left.4^{\triangle I E C}, n=6-8\right)$ mice aged 8-12 weeks were housed in ventilated cages in groups of 3-6 ani- 
Table 1. Real-time PCR primer sequences for TLR4 and $\beta$-actin

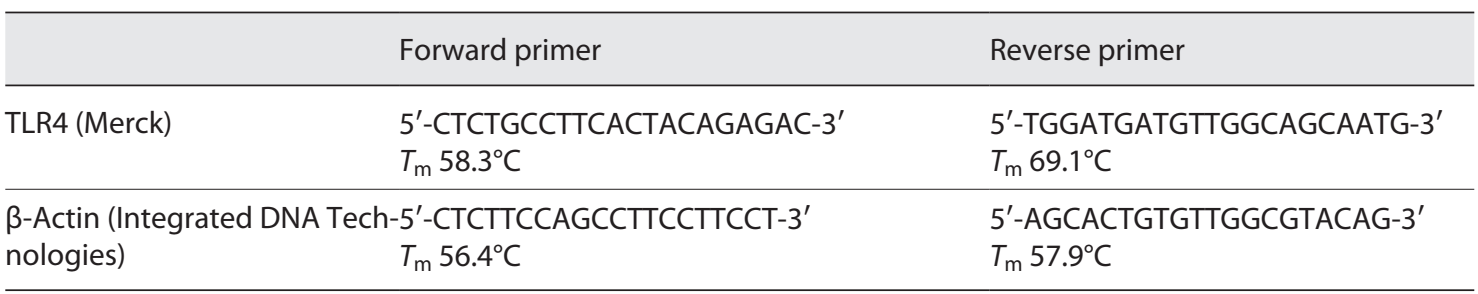

TLR4, Toll-like receptor 4; PCR, polymerase chain reaction.

mals per cage with a 12-hour light/dark cycle and access to irradiated standard mouse chow and sterile water. Mice were euthanized via $\mathrm{CO}_{2}$ exposure and cervical dislocation prior to dissection, in accordance with ethical approval of the University of Adelaide Animal Ethics Committee (M-2019-020) and the University of Adelaide Institutional Biosafety Committee (IBC approval number 14254). The study complied with the National Health and Medical Research Council (Australia) Code of Practice for Animal Care in Research and Teaching (2014).

\section{Breeding Strategy and Genetic Confirmation}

The intestinal epithelial conditional TLR4 KO C57BL/6 $\left(T l r 4^{\triangle I E C}\right)$ mouse model was created by following a transgenic Vil1-cre/Tlr4loxP breeding strategy (The Jackson Laboratory, Bar Harbor, ME, USA). By crossing a homozygous Tlr4loxP/Vil1-cre WT with a homozygous Tlr4loxP/hemizygous Vill-cre, this breeding strategy resulted in 1 in 2 offspring being the desired conditional KO. Conditional KO of TLR4 was confirmed via polymerase chain reaction (PCR) analysis as per protocols provided by the Jackson Laboratory [13] for TLR4flox [14] and Vilcre genes [15], where DNA was extracted from mouse ear notches using the $\mathrm{Nu}$ cleospin Tissue DNA extraction kit and used at a working concentration of $20 \mathrm{ng} / \mu \mathrm{L}$ (Machery-Nagel, Duren, Germany). Primer sequences used for confirmation of genotype were as follows: Vilcre forward GCTTTCAAGTTTCATCCATGTTG, Vil-cre WT reverse TTCATGATAGACAGATGAACACAGT, Vil-cre mutant reverse GTCTTTGGGTAAAGCCAAGC, TLR4-floxed forward TGACCACCCATATTGCCTATAC, and TLR4-floxed reverse TGATGGTGTGAGCAGGAGAG. Cycling conditions for Vil-cre were as follows: denaturing at $95^{\circ} \mathrm{C}$ for $3 \mathrm{~min}$, then $95^{\circ} \mathrm{C}$ for $5 \mathrm{~s}$, and then $60^{\circ} \mathrm{C}$ for $30 \mathrm{~s}$. The final 2 steps were repeated for 40 cycles. The mutant band, representing the presence of hemizygous Vilcre was at 85 base pairs, compared to WT Vil-cre at 119 base pairs. Cycling conditions for TLR4 flox were $94^{\circ} \mathrm{C}$ for $2 \mathrm{~min}$, then the following steps were repeated for 10 cycles: $94^{\circ} \mathrm{C}$ for $20 \mathrm{~s}, 65^{\circ} \mathrm{C}$ for $15 \mathrm{~s}$ (decreasing by $0.5^{\circ} \mathrm{C}$ each cycle), and $68^{\circ} \mathrm{C}$ for $10 \mathrm{~s}$. Following from this, samples were cycled 28 times at $94^{\circ} \mathrm{C}$ for $16 \mathrm{~s}, 60^{\circ} \mathrm{C}$ for $15 \mathrm{~s}$, and then $72^{\circ} \mathrm{C}$ for $10 \mathrm{~s}$. The final step was $2 \mathrm{~min}$ at $72^{\circ} \mathrm{C}$. Homozygous TLR4-floxed samples produced a band of 285 base pairs, heterozygous samples were at 234 base pairs and 285 base pairs, and WT TLR4 produces a band at 234 base pairs. For visualizations, samples were run in $4 \%$ agarose and visualized using Midori Green Advance DNA stain (Nippon Genetics, Tokyo, Japan). Conditional KO of intestinal epithelial TLR4 $\left(T l r 4^{\Delta I E C}\right)$ resulted in PCR showing mutant Vil-cre ( 85 base pairs) and homozygous TLR4-floxed (285 base pairs).

Intestinal Characterization of Intestinal Epithelial TLR4 Knockout
Further confirmation of successful $\mathrm{KO}$ was conducted by realtime PCR, where small intestinal tissue for both WT and Tlr $4^{\Delta I E C}$ was harvested and scraped to separate the submucosal layer for epithelial-specific TLR4 analyses. For both strains, the whole small intestine was dissected and opened longitudinally. Using a rounded scalpel, a light feather-like scraping was undertaken to separate out an epithelial-dominant sample. Ten nanograms of RNA extracted from these scrapings for both WT and Tlr $4^{\triangle I E C}$ mice was reverse transcribed using the iScript cDNA Synthesis Kit (\#1708890; BioRad, Gladesville, NSW, Australia) as per manufacturer's instructions. RT-PCR was performed using the Rotor-Gene 3000 (Corbett Research Sydney, Mortlake, NSW, Australia). Amplification mixes contained $1 \mu \mathrm{L}$ of cDNA sample $(100 \mathrm{ng} / \mu \mathrm{L}), 5 \mu \mathrm{L}$ of SYBR green fluorescence dye (QuantiTect; Qiagen, Hilden, Germany), $3 \mu \mathrm{L}$ of RNase-free water (Macherey Nagal, Duren, Germany), and $0.5 \mu \mathrm{L}$ of each forward and reverse primers $(50 \mathrm{pmol} / \mu \mathrm{L})$, to make a total reaction volume of $10 \mu \mathrm{L}$. Primer details are as presented in Table 1. Thermal cycling conditions were: $95^{\circ} \mathrm{C}$ for $10 \mathrm{~min}, 40$ cycles of $95^{\circ} \mathrm{C}$ for $10 \mathrm{~s}, 59^{\circ} \mathrm{C}$ for $30 \mathrm{~s}$, and $72^{\circ} \mathrm{C}$ for $45 \mathrm{~s}$ and a final melt step of $60-95^{\circ} \mathrm{C}$ changing $1^{\circ} \mathrm{C}$ per step, holding for $5 \mathrm{~s}$ each. Samples were run in triplicate, including negative controls (no cDNA template). Experimental threshold (CT) values were calculated by the Rotor Gene 6 programme. CT values were used to quantify relative mRNA expression of TLR4 and $\beta$-actin using the $\Delta C_{T}$ method, where relative expression $=2^{-(\text {CT TLR } 4-\text { CT } \beta \text {-actin })}[16]$.

\section{Ex vivo Electrophysical Assessments}

Ussing chambers (EM-CSYS-8 with DM-MC8 voltage clamp/ electrode input; Physiologic Instruments, San Diego, CA, USA) were used to assess intestinal electrophysiology in WT and Tlr $4^{\Delta I E C}$ mice as previously described [16]. Briefly, segments of ileum and colon were dissected from mice and flushed with ice-cold $1 \times$ phosphate-buffered saline (PBS). One $\mathrm{cm}$ segments were opened longitudinally along the mesenteric attachment line, mounted into 0.1 $\mathrm{cm}^{2}$ aperture sliders (P2303A; Physiologic Instruments), and inserted into chambers filled with a glucose-fortified Ringers solution consisting of (in millimolar): $\mathrm{NaCl} 115.4, \mathrm{KCl} 5, \mathrm{MgCl}_{2} 1.2, \mathrm{NaH}_{2} \mathrm{PO}_{4}$ $0.6, \mathrm{NaHCO}_{3} 25, \mathrm{CaCl}_{2} 1.2$, and glucose 10 , bubbled with carbogen gas $\left(95 \% \mathrm{O}_{2}, 5 \% \mathrm{CO}_{2}\right)$ and warmed to $37^{\circ} \mathrm{C}$ [16]. Ileal segments had the mucosal side bathed in mannitol-fortified Ringers $(10 \mathrm{mM})$ to maintain osmotic balance. Once mounted, tissue was voltageclamped to zero potential difference, establishing baseline readings. Tissue was allowed to equilibrate for $20 \mathrm{~min}$ before short circuit current (Isc, marker of net ion transport/secretion), and transepithelial electrical resistance (marker of barrier integrity) was measured using Acquire and Analyse Revision II (v2.3; Physiologic Instruments). 
Histopathological Analyses

Hematoxylin and Eosin Staining

Mouse ileum and colon samples were fixed in $10 \%$ formalin and embedded into paraffin wax blocks. Formalin-fixed paraffin embedded blocks were sectioned $(4 \mu \mathrm{m})$ and mounted on SuperFrost White slides (Menzel-Gläser, Braunschweig, Germany). Slides were then fixed on a $37^{\circ} \mathrm{C}$ heat block, for a minimum of $1 \mathrm{~h}$. Standard hematoxylin and eosin staining procedures were followed [6]. In brief, slides were dewaxed in $3 \times$ washes in $100 \%$ histolene for 5 min each and then rehydrated with graded ethanol as previously described [6]. Slides were then stained in Lille-Mayers hematoxylin for $5 \mathrm{~min}$ and rinsed until clear in running tap water. Slides were then quickly dipped twice in $1 \%$ acid alcohol $(5 \mathrm{~mL} \mathrm{HCl}+500 \mathrm{~mL}$ $70 \%$ ethanol) and washed in running tap water until clear. Tissue was then placed in Scott's Tap Water (in millimolar) $\left(\mathrm{MgSO}_{4}\right.$ 166.2; $\mathrm{NaHCO}_{3} 23.7$ in $1 \mathrm{~L} \mathrm{dH}_{2} \mathrm{O}$ ) for 2 min and washed. Counterstaining with alcoholic eosin (Sigma-Aldrich, St Louis, MO, USA) occurred for $2 \mathrm{~min}$, and slides were washed with running tap water until clear. Slides were then treated with $90 \%$ ethanol $(30 \mathrm{~s})$ and $100 \%$ ethanol (30 s) and finally cleared with $3 \times$ washes in $100 \%$ histolene for $5 \mathrm{~min}$ each. Slides were coverslipped with D.P.X neutral mounting medium (Sigma-Aldrich).

Slides were imaged using an Olympus BX51 light microscope and Olympus DP20 microscope camera (Olympus, Tokyo, Japan). A modified version of well-established intestinal injury scoring criteria [17] was used to quantify possible differences between groups, with 1 representing the presence and 0 representing the absence of the pathophysiological marker. The criteria included: disruption of brush border and surface enterocytes, crypt loss/architectural disruption, disruption of crypt cells, infiltration of polymorphonuclear cells and lymphocytes, dilation of lymphatics and capillaries, edema, villous fusion, and villous atrophy. The latter 2 criteria were not assessed in colon samples; therefore, the total possible score for ileum samples was 8 and for colon samples was 6 . Furthermore, ileum villus height and crypt depth and colonic mucosal thickness were assessed by a blinded researcher (E.E.B.) using the Olympus cellSens Standard imaging program (Olympus).

\section{Alcian Blue and Periodic Acid-Schiff}

Alcian blue and periodic acid-Schiff was used to quantify goblet cells in the mucosal of the ileum and colon from formalin-fixed paraffin-embedded tissue blocks. Tissue was cut into $4-\mu \mathrm{m}$ sections and placed on SuperFrost White slides (Menzel-Gläser). Slides were dewaxed in $3 \times$ washes in $100 \%$ histolene for 5 min each and then rehydrated with graded ethanol. Slides were stained in

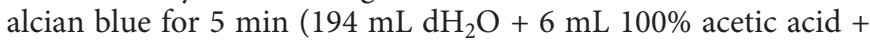
$2 \mathrm{~g}$ alcian blue) before being incubated in $0.5 \%$ periodic acid for 5 min. Slides were again washed with $\mathrm{dH}_{2} \mathrm{O}$ and then incubated in Schiff's reagent for $15 \mathrm{~min}$ before being counterstained with hematoxylin for $30 \mathrm{~s}$. Slides were cleared in $100 \%$ histolene $(3 \times 5 \mathrm{~min})$ and coverslipped with D.P.X neutral mounting medium. Slides were imaged using the Nanozoomer Digital slide scanner, with goblet cell counts conducted by a blinded researcher (K.R.S.).

\section{Immunofluorescence of Tight Junction Proteins and Immune} Cells

To investigate molecular determinants of barrier function, immunofluorescence for ZO-1 and occludin was performed. Briefly, the intestine was removed and immediately flushed with ice-cold

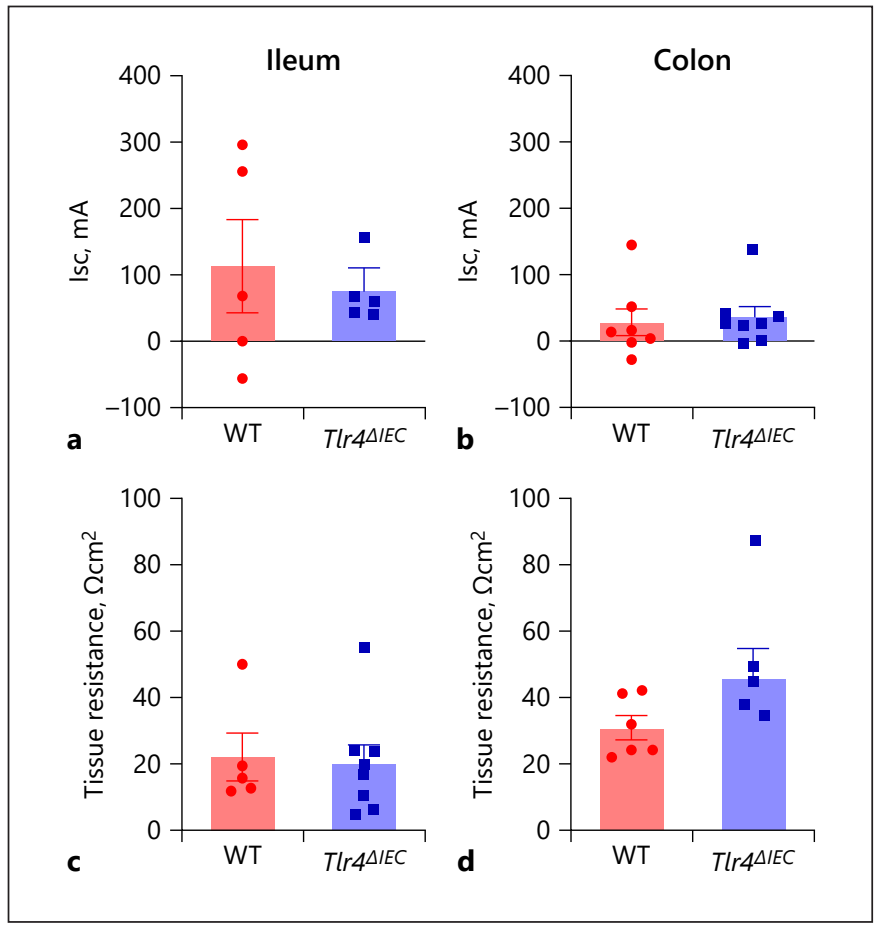

Fig. 1. Intestinal electrophysiology is not dependent on TLR4 expression. Baseline short-circuit current (Isc, mA) for the ileum (a) and colon (b) and transepithelial tissue resistance $\left(\Omega \times \mathrm{cm}^{2}\right)$ for the ileum $(\mathbf{c})$ and colon $(\mathbf{d})$ samples in WT and Tlr $4^{\Delta I E C}$ mice. No difference between groups (WT $n=5-7$ and $T l r 4^{\Delta I E C} n=6-8, p>$ $0.05)$. Data presented as mean \pm SEM. WT, wild-type; SEM, standard error of mean; TLR4, Toll-like receptor 4.

$1 \times$ PBS. Segments of the ileum and colon were fixed in $10 \%$ neutral buffered formalin, processed, and embedded into paraffin wax. Tissues were then cut into $4-\mu \mathrm{m}$ sections and placed onto FLEX IHC microscope slides (Flex Plus Detection System, \#K8020; Dako, Næstved, Denmark) and heated on a heat pad. Slides were deparaffinised via $3 \times$ washes with $100 \%$ histolene and rehydrated with graded ethanol (100\% ethanol for $30 \mathrm{~s}, 90 \%$ ethanol for $30 \mathrm{~s}$ and $70 \%$ ethanol for $30 \mathrm{~s}$ ) [16]. Antigen retrieval was via the PT Link bath (pre-treatment module, \#PT101; Dako) using an EDTA/ Tris buffer consisting of (in millimolar): Tris 9.9 and EDTA 1.3 and $0.5 \mathrm{~mL}$ Tween 20 in $1.5 \mathrm{~L} \mathrm{dH}_{2} \mathrm{O}, \mathrm{pH}=9$ at $97^{\circ} \mathrm{C}$ for $20 \mathrm{~min}$.

Tissue samples were stained using the DakoCytomation Autostainer (AutostainerPlusTM; Dako, serial number: AS1271F1104). The primary antibodies used were as follows: ZO-1 (61-7300; Invitrogen, Carlsbad, CA, USA, $0.25 \mathrm{mg} / \mathrm{mL}, 1: 100$ dilution), occludin (33-1500; Invitrogen, $0.5 \mathrm{mg} / \mathrm{mL}, 1: 200$ dilution), and CD11b (ab133357; Abcam, Cambridge, UK, 1:1,000 dilution). Primary antibody was diluted in 5\% normal horse serum (Sigma-Aldrich), $1 \times$ PBS for tight junction analyses and $1 \%$ bovine serum albumin (BSA) for immune cell analyses (Sigma-Aldrich). The secondary antibodies were AlexaFluor 488 anti-mouse (occludin), AlexaFluor 488 anti-rabbit (CD11b), and AlexaFluor 568 anti-rabbit (ZO1) (Thermo Fisher, Waltham, MA, USA). The secondary antibody was diluted in $1 \times$ PBS, $1 \%$ BSA, and $2 \%$ fetal bovine serum. DAPI $(1 \mu \mathrm{g} / \mathrm{mL})$ (Sigma-Aldrich) counterstaining was utilized to visual- 
Fig. 2. Epithelial TLR4 deletion does not influence ZO-1 expression. ZO-1 expression (red) in WT and Tlr $4^{\triangle I E C}$ mice ileum and colon with DAPI (blue) counterstain of nuclei; WT ileum (a), Tlr4 ${ }^{\triangle I E C}$ ileum (b), WT colon (c), Tlr $4^{\triangle I E C}$ colon (d), magnification $\times 40$. No difference in ZO- 1 expression for either (e) ileum or (f) colon ( $\%$ area stain, $n=6, p>0.05)$. Data presented as mean \pm SEM. ZO-1, zonular occludin 1; WT, wild-type; SEM, standard error of mean; TLR4, Toll-like receptor 4 .

Fig. 3. Occludin expression is independent of epithelial TLR4 expression. Occludin expression (green) in WT and Tlr $4^{\triangle I E C}$ mice ileum and colon with DAPI (blue) counterstain of nuclei; WT ileum (a), X ileum (b), WT colon (c), Tlr $4^{\triangle I E C}$ colon (d), magnification $\times 40$. No difference in occludin expression for either (e) ileum or (f) colon (\% area stain, $n=6, p>0.05)$. Data presented as mean \pm SEM. WT, wild-type; SEM, standard error of mean; TLR4, Toll-like receptor 4 .
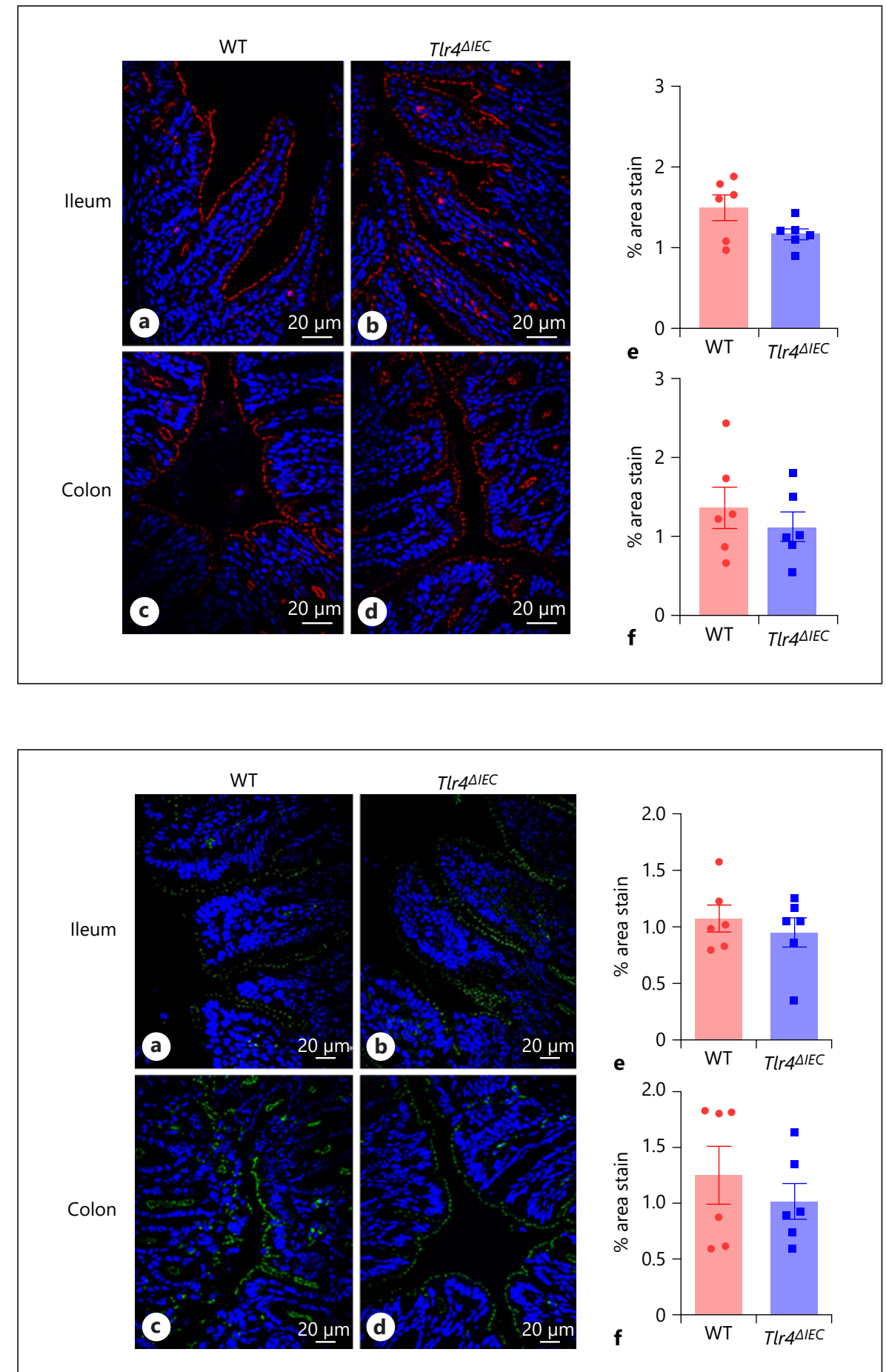

ize the nucleus of cells in sample, with $1 \times$ PBS as the diluent. A protein block of $10 \%$ normal horse serum for tight junctions and $4 \%$ BSA for immune cells was used to reduce nonspecific antibody binding during the procedure.

Post-staining, a drop of Fluoroshield (Sigma-Aldrich) was applied to each slide and coverslipped. Slides were then stored in the dark at $4^{\circ} \mathrm{C}$ to await imaging. Slides were imaged using the Nikon

Intestinal Characterization of Intestinal Epithelial TLR4 Knockout
A1 Confocal Microscope using a $\times 40$ objective. Fluorescent staining was quantified via \% area stain on the Fiji Image J program as previously described [18].

\section{Statistics}

All data were compared using Prism version 8.0 (GraphPad Software, San Diego, CA, USA). Data were first assessed was for 
normality using the Shapiro-Wilk test. Parametric data were analyzed using a one-way ANOVA or $t$ test and presented as mean \pm standard error of mean (SEM). Nonparametric data were analyzed using a Kruskal-Wallis test and presented as median and range. $p$ values $<0.05$ were deemed significant.

\section{Results}

Epithelial TLR4 Does Not Control Intestinal Barrier

Function, Tight Junction Integrity, or Immune Cell

Infiltration in Healthy Mice

Successful conditional KO of TLR4 on intestinal epithelial cells was confirmed by both genotyping and RTPCR analysis, with 8-fold higher TLR4 expression in WT intestinal epithelial-dominant scrapings than in Tlr $4^{\Delta I E C}$ scrapings (mean \pm SEM): WT $0.29 \pm 0.02$ versus Tlr $4^{\Delta I E C}$ $0.04 \pm 0.02, p=0.012$.

No difference between WT and $T l r 4^{\triangle I E C}$ was observed in the ileum or colon in baseline short-circuit current: ileum (mean \pm SEM): WT $113.3 \pm 69.8$ versus $T l r 4^{\triangle I E C}$ $75.3 \pm 34.9 \mathrm{~mA} p=0.607$, colon: WT $28.4 \pm 21.1$ versus $T l r 4^{\Delta I E C} 36.7 \pm 15.5 \mathrm{~mA} p=0.752$ (Fig. 1a, b). Similarly, there was no difference in baseline transepithelial tissue resistance: ileum (mean \pm SEM): WT $22 \pm 7.2$ versus $T l r 4^{\Delta I E C} 20 \pm 5.6 \Omega \times \mathrm{cm}^{2}, p=0.831$, colon WT $30.8 \pm 3.6$ versus $\operatorname{Tl} 4^{\triangle I E C} 45.1 \pm 9.5 \Omega \times \mathrm{cm}^{2}, p=0.191$ (Fig. 1c, d).

ZO-1 and occludin staining was evident at the apicolateral border of epithelial cells of villous and crypt structures of the ileum and colon (Fig. 2, 3). Quantification of tight junction protein staining ( $\%$ area stain) showed no differences for ZO-1: ileum (mean \pm SEM) WT $1.49 \pm$ 0.155 versus $T l r 4^{\Delta I E C} 1.17 \pm 0.07 p=0.09$; colon WT 1.36 \pm 0.26 versus $T l r 4^{\Delta I E C} 1.12 \pm 0.18 p=0.47$. Similarly, there was no difference in occludin expression: ileum (mean \pm SEM) WT $1.07 \pm 0.12$ versus $T l r 4^{\Delta I E C} 0.95 \pm 0.13 p=0.53$; colon WT $1.26 \pm 0.26$ versus Tlr $4^{\Delta I E C} 1.02 \pm 0.16 p=0.45$. Positive CD11b staining (Fig. 4) was evident in both the ileum mucosa and colon mucosa and submucosa. CD11bpositive immune cells in the ileum were mildly decreased in WT mice: (\% area stain, mean \pm SEM) WT $0.14 \pm 0.02$ versus $T l r 4^{\triangle I E C} 0.09 \pm 0.01 p=0.04$. However, there were no differences in CD11b-positive immune cells in the colon between strains: ( $\%$ area stain, mean \pm SEM) WT $0.53 \pm 0.08$ versus $T l r 4^{\Delta I E C} 0.49 \pm 0.08, p=0.73$.

\section{Epithelial TLR4 Deletion Does Not Affect Intestinal Morphometry}

No histological differences were observed between WT and Tlr $4^{\triangle I E C}$ mice in the ileum or colon (Fig. 5a-d), with no change in villus height $(p=0.49)$, ileum crypt depth $(p=0.66)$, or colonic crypt depth $(p=0.52)$ (Fig. 5e$\mathrm{g}, p>0.05)$. Furthermore, to ensure a comprehensive and translational assessment of intestinal structure, a tissue injury score was assessed. No evidence of microscopic injury was detected in either WT or Tlr ${ }^{\Delta I E C}$ ileum or colon tissue (ileum $p=0.617$, colon $p=0.529$ ). Finally, no difference in goblet cell abundance in ileum villi, ileum crypt, and colon crypt between WT and Tlr ${ }^{4 I E C}$ was observed (Fig. 6a-g, $p>0.05$ for all groups).

\section{Discussion/Conclusion}

TLR4 has received a significant amount of attention for its homeostatic control and therapeutic applications based on its assumed regulation of mucosal barrier function. This is the first study to present the baseline intestinal characteristics of epithelial-specific TLR4 KO mice $\left(T l r 4^{\triangle I E C}\right)$ and has found that contrary to expectations, the absence of epithelial TLR4 did not alter intestinal homeostasis. These data highlight the importance of sitespecific TLR4 investigation and underscore the limitations of extrapolating evidence from disease models to healthy states.

The concept that TLR4 is involved in homeostatic control is clearly outlined in studies investigating the nervous control of intestinal tissue $[19,20]$ and immune tolerance [21]. However, TLR4 involvement in homeostatic control of barrier function has been inferred from disease modeling studies. Shi et al. [22] found that intestinal injury in response to dextran sulfate sodium induced colitis was significantly aggravated in a global TLR4 KO mouse model. This suggests TLR4 expression is a protective component of the intestines and supports the healthy functioning of the intestinal barrier [22]; however, this is contradictory to earlier findings which show that TLR4 overexpression leads to impaired intestinal epithelial cell differentiation and barrier dysfunction [23]. Since not all studies distinguish between site-specific expression of TLR4, contradicting evidence is expected. Our study is one of the first to entirely focus on how intestinal epithelial TLR4 expression influences the healthy state of the intestines.

As outlined, our data suggest that epithelial TLR4 is not essential to the regulation of the intestinal environment, namely the role of tight junction protein expression, goblet cell populations, and functional tissue permeability in healthy development. While minor nonsignificant differences have been noted between groups, this 
Fig. 4. Immune cell infiltration does not depend on epithelial TLR4 expression. CD11b expression (green) in WT and $T l r 4^{\Delta I E C}$ mice ileum and colon with DAPI (blue) counterstain of nuclei; WT ileum (a), Tlr $4^{\Delta I E C}$ ileum (b), WT colon (c), Tlr $4^{\triangle I E C}$ colon (d), magnification $\times 40$. Difference in CD11b immune cells in (e) ileum $\left({ }^{*} p=0.04\right)$, no difference in (f) colon (\% area stain, $n=6, p>0.05$ ). Data presented as mean \pm SEM. WT, wild-type; SEM, standard error of mean; TLR4, Tolllike receptor 4 .
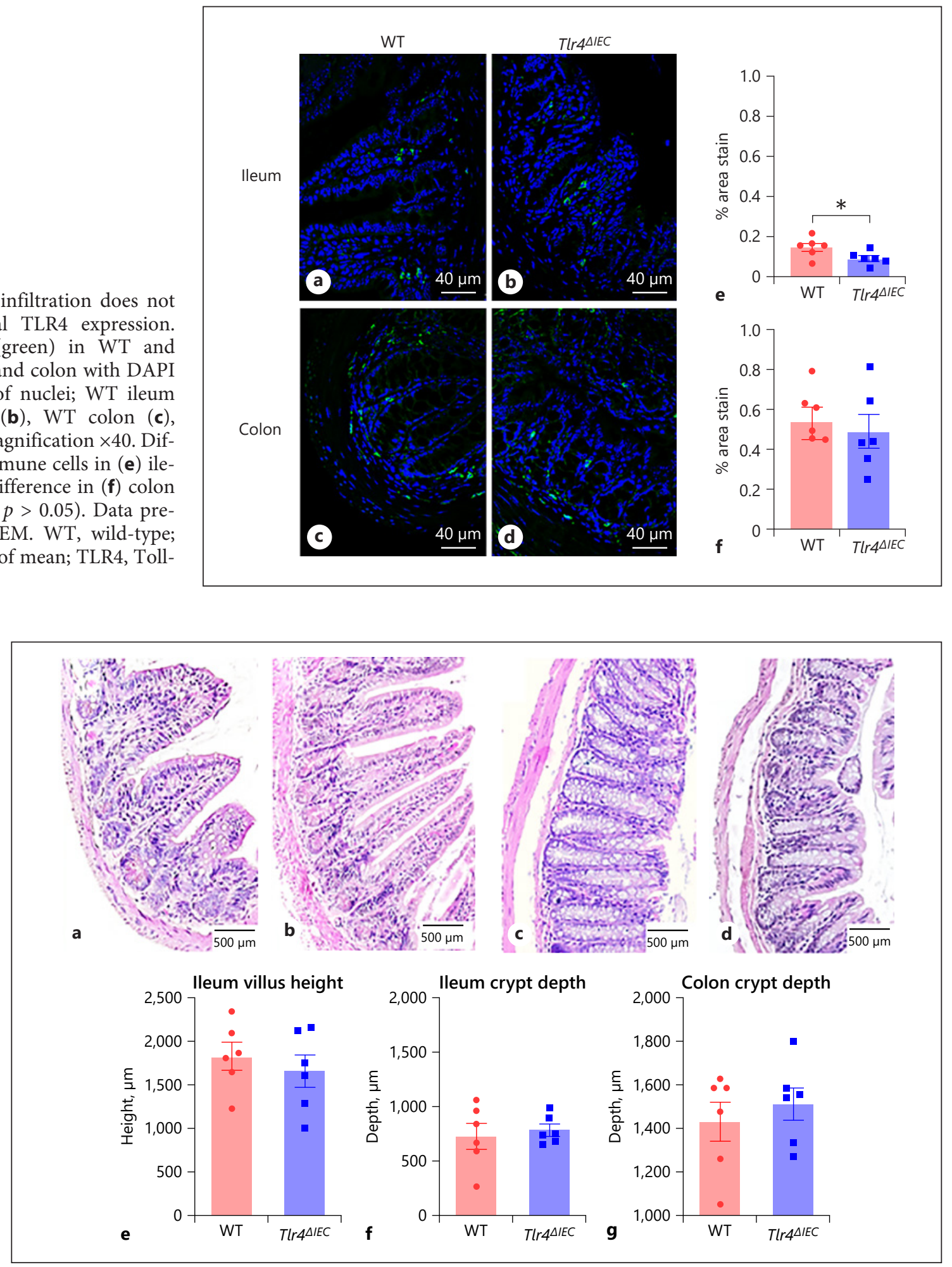

Fig. 5. Epithelial TLR4 deletion does not affect intestinal architecture. H\&E stain of the ileum and colon from WT and Tlr ${ }^{\triangle I E C}$ mice. WT ileum (a), Tlr $4^{\triangle I E C}$ ileum (b), WT colon (c), Tlr $4^{\triangle I E C}$ colon (d), magnification $\times 20$. No difference in ileum villus height $(\mathbf{e}, \mu \mathrm{m})$, ileum crypt depth $(\mathbf{f}, \mu \mathrm{m})$, or colon crypt depth $(\mathbf{g}, \mu \mathrm{m})(n=6, p>$ 0.05). Data presented as mean \pm SEM. H\&E, hematoxylin and eosin; WT, wild-type; SEM, standard error of mean; TLR4, Toll-like receptor 4 .
Intestinal Characterization of Intestinal Epithelial TLR4 Knockout
Inflamm Intest Dis 2021;6:199-209 DOI: $10.1159 / 000519200$ 


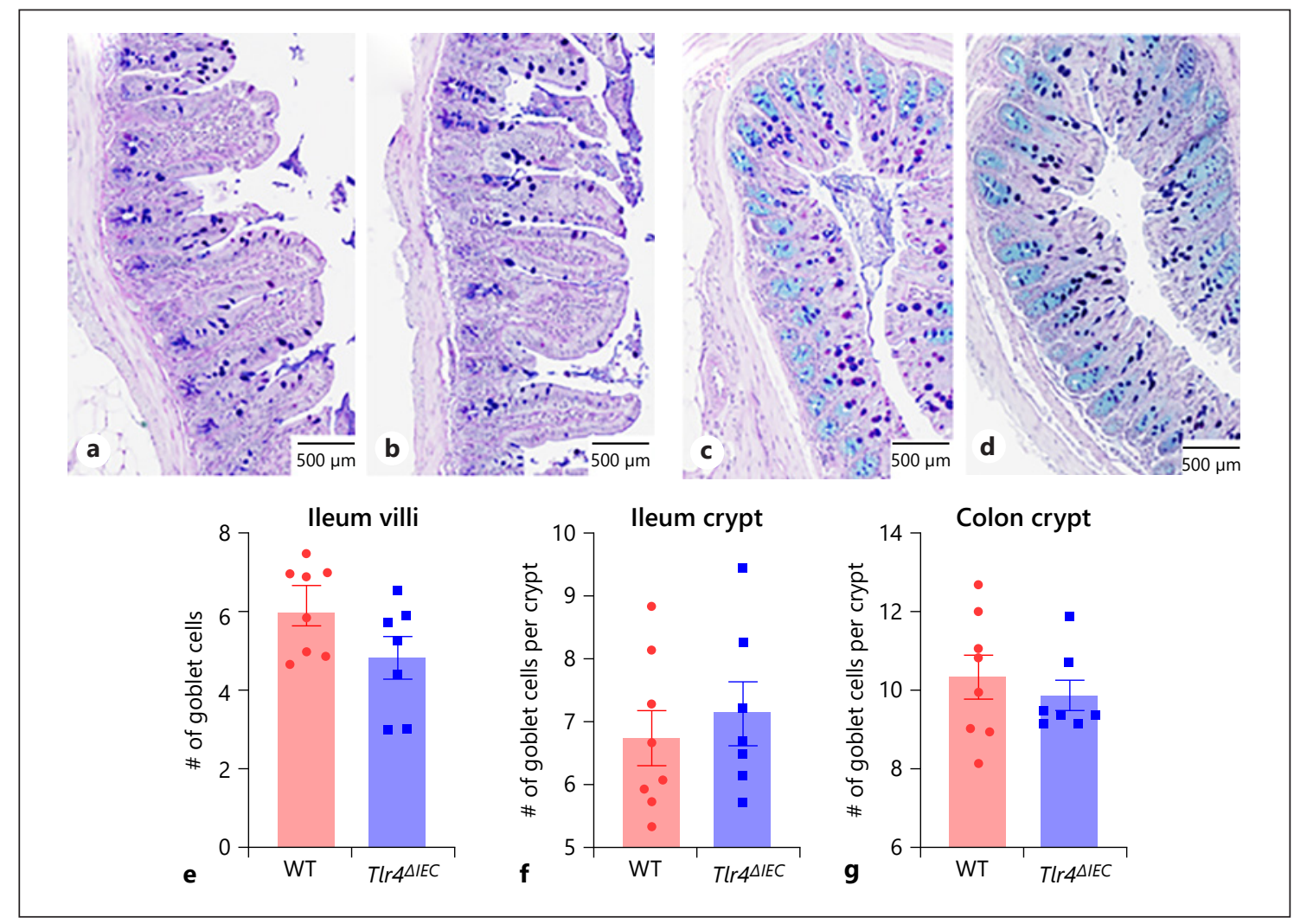

Fig. 6. Goblet cell abundance is not affected by TLR4 expression in the intestinal epithelium. AB-PAS stain of the ileum and colon from WT and Tlr $4^{\Delta I E C}$ mice to visualize goblet cells (dark purple/blue stain). WT ileum (a), $T l r 4^{\triangle I E C}$ ileum (b), WT colon (c), Tlr $4^{\Delta I E C}$ colon (d), magnification $\times 20$. No differences in goblet cells in ileum villi (e), ileum crypt (f) or colon crypts (g) (\# of goblet cells per structure, WT $n=8$, Tlr $4^{\Delta I E C} n=7, p>0.05$ ). Data presented as mean \pm SEM. AB-PAS, alcian blue and periodic acid-Schiff; WT, wild-type; SEM, standard error of mean; TLR4, Toll-like receptor 4 .

is most likely due to stochastic variation. Previous research conducted by our group has demonstrated intestinal permeability and morphology changes in a positive control of epithelial tissue disruption. In mice treated with the chemotherapy irinotecan, baseline intestinal conductance (a measure of intestinal permeability, the opposite of tissue resistance) was significantly increased $\left(53.19 \pm 6.46 \mathrm{~s} / \mathrm{cm}^{2},+105.62 \%\right.$ relative to WT controls; $p=0.0008$ ) [16]. Furthermore, irinotecan-treated small intestinal and colonic tissue showed severe damage, including villous blunting and crypt degeneration [24]. There is a substantial difference between these positivecontrol outcomes and the minor changes in the present study, therefore suggesting that current minor variability in the Tlr $4^{\triangle I E C}$ versus WT data is inconsequential. Our current findings indicate that there may be compensatory mechanisms controlling the gastrointestinal microenvironment in the absence of epithelial TLR4. A possible mechanism could be that immune TLR4 is responsible for modulating barrier function and intestinal homeostasis. This aligns with the higher TLR4 expression on immune cell populations and acknowledges the profound immune infiltrate of the gut [25]. TLR4 is expressed on a range of immune cells including, macrophages, myeloid cells, and dendritic cells [3] and has proven roles in dendritic cell maturation [26] and immune tolerance [27]. Considering that immune TLR4 has been shown to control immune system functioning and development of a healthy microbiome, it could be also deduced that immune TLR4 aids in controlling intestinal permeability and barrier function in mice. This notion is supported by our data, which showed no difference in intestinal characteristics between the Tlr $4^{\triangle I E C}$ and WT mice. To confirm this role of exclusive immune TLR4 signaling, future work could be conducted in conditional mice where there is a deletion of immune TLR4 expression. 
An alternative mechanism possibly responsible for this compensation could include the recognition of pathogens and tolerance of the commensal microbiota via different TLRs, notably TLR2 [8]. Upon ligand binding, TLR2 activates the MyD88-dependent inflammatory pathway [28]. This pathway is also activated in response to TLR4 activation; therefore, a distinct overlap in TLR4 and TLR2 signaling exists [28]. The similarity between TLR2 and TLR4 is best shown in healthy states, with an early study finding that activation of TLR2 and TLR4 primes dendritic cell tolerance to commensal organisms [29]. Furthermore, the combination of TLR2 and TLR4 signaling is indicated in the healthy control of spontaneous and serotonin-induced contractile responses of mouse ileum [20]. It is the commonality between TLR2 and TLR4 signaling pathways, which may explain why intestinal homeostasis was maintained in our Tlr4 $4^{\triangle I E C}$ model. A previous research study has investigated disruption of TLR pathways, including TLR2 KO, not MD-2, and has shown in intestinal models of chemotherapy-induced intestinal mucositis that deletion of TLR2 alone increased intestinal inflammation and damage, suggesting TLR2 is a potential therapeutic target [30]. While this evidence indicates importance of TLR2, rather than TLR4, in intestinal regulation, unfortunately, epithelial deletion of TLR2 has not been previously studied in either healthy or diseased states. Therefore, future research could be centered on a TLR2 epithelial-specific KO mouse model, to further investigate this complex relationship between different TLR expression and intestinal function.

Overall, our findings support the use of this $T l r 4^{\Delta I E C}$ mouse line in the investigation of gastrointestinal disease, where TLR4 may be of interest. These mice showed no difference in baseline intestinal characteristics compared to WT, therefore displaying no inherent variability of intestinal function caused by genetic modification of intestinal epithelial TLR4. This is a promising sign for the ongoing viability of this model as the retention of normal intestinal function suggests that the Tlr $4^{\triangle I E C}$ model is reliable. This could allow for future disease models in the $T l r 4^{\Delta I E C}$ mice to dissect the contribution of epithelial TLR4 to disease development. Translationally, the use of these $T l r 4^{\Delta I E C}$ mice in models of gastrointestinal disease will provide much greater insight into the site-specific contribution of TLR4. This would allow for the guiding of future therapeutics, including nanoparticle delivery systems allowing epithelial TLR4 to be augmented in a manner that prevents any systemic effect [31]. This could possibly include TLR4 agonist or antagonist delivery to the site-specific area, meaning that only the intestinal epithelial population of TLR4 would be altered, leaving immune and nervous TLR4 functioning uninterrupted. This is especially important where site-specific TLR4 expression shows distinct and potentially contradicting mechanisms, for example, following cancer treatments $[6,32]$. However, while these results shed further insight into the mechanistic roles of epithelial TLR4, they should be approached with caution as this study utilized a small sample size where further functional data, such as metabolic and absorptive capacity, were not assessed. A further limitation of the current study is the exclusion of other related $\mathrm{KO}$ models, such as immune-specific TLR4 or TLR2 KO. As data presented in the Tlr $4^{\Delta I E C}$ mice revealed no differences in intestinal functioning, it can be deduced that epithelial TLR4 is likely to have a minor role in intestinal homeostasis. Consequently, future research including these alternative $\mathrm{KO}$ models would greatly enhance this field of knowledge.

In conclusion, TLR4 is an important immunosurveillance protein to many areas of current medical research, including inflammatory gastrointestinal diseases and chemotherapy-induced gastrointestinal toxicity $[5,6]$. While there is a large body of research surrounding the dual roles of TLR4 in both healthy states and disease, currently, there is very little distinction of cell-specificity in research outcomes. This oversight has the potential to influence the translation of results to clinical practice. To facilitate the emergence of research that considers cellspecific TLR4 expression, a well-validated intestinal epithelial TLR4 conditional mouse model ( $\left.T l r 4^{\Delta I E C}\right)$ must exist. The current study verified that $T l r 4^{\triangle I E C}$ mice are not fundamentally altered prior to future disease modeling studies. These results both support the use of this model in future studies and has presented novel insights into the role of intestinal epithelial TLR4 in homeostatic control.

\section{Acknowledgment}

We would like to acknowledge the Molecular Diagnostic Solutions Group with CSIRO Health \& Biosecurity, Adelaide, SA, Australia, for training and use of the Nikon A1 Confocal Microscope. Further, we would like to acknowledge the University of Adelaide, Adelaide Microscopy and the Australian Microscopy and Microanalysis Research Facility (AMMRF). Finally, we would also like to acknowledge Laboratory Animal Services with the University of Adelaide, Australia for continued support, training, and care of animals. This research is supported by an Australian Government Research Training Program (RTP) Scholarship. 


\section{Statement of Ethics}

The study design was approved by the Animal Ethics Committee of the University of Adelaide and complied with the National Health and Medical Research Council (Australia) Code of Practice for Animal Care in Research and Teaching (2014) (animal ethics approval No.: M-2019-020).

\section{Conflict of Interest Statement}

The authors have no conflicts of interest to declare.

\section{Funding Sources}

H.R.W. was funded by the National Health and Medical Research Council CJ Martin Biomedical Research Fellowship, (20182022). E.E.C. was funded by the Australian Government RTP Scholarship, (2019-2022). K.R.S. was funded by the Australian Government RTP Scholarship (2017-2021) and Lion's Medical Research Foundation Scholarship (2017-2020).

\section{Author Contributions}

All the authors fulfill the ICMJE criteria for authorship. Elise E. Crame is the primary researcher associated with this research and substantially contributed to research design, data acquisition, analysis, and interpretation. She is the primary author of this research, including the drafting and revising process. Joanne $\mathrm{M}$. Bowen, Janet K. Coller, and Hannah R. Wardill provided significant contribution to the study design and conception and had substantial involvement in writing, drafting, and the revision process. Kate R. Secombe provided substantial contribution to data acquisition, analysis, and interpretation. Maxime François and Wayne Leifert provided substantial contribution to data acquisition, analysis, and interpretation.

\section{Data Availability Statement}

All data generated or analyzed during this study are included in this article. Further inquiries can be directed to the corresponding author.

\section{References}

1 Pott J, Hornef M. Innate immune signalling at the intestinal epithelium in homeostasis and disease. EMBO Rep. 2012;13(8):684-98.

2 Wells JM, Brummer RJ, Derrien M, MacDonald TT, Troost F, Cani PD, et al. Homeostasis of the gut barrier and potential biomarkers. Am J Physiol Gastrointest Liver Physiol. 2017; 312(3):G171-93.

3 Vaure C, Liu Y. A comparative review of tolllike receptor 4 expression and functionality in different animal species. Front Immunol. 2014:5:316.

4 McClure R, Massari P. TLR-dependent human mucosal epithelial cell responses to microbial pathogens. Front Immunol. 2014;5: 386.

5 Belmonte L, Beutheu Youmba S, BertiauxVandaële N, Antonietti M, Lecleire S, Zalar A, et al. Role of toll like receptors in irritable bowel syndrome: differential mucosal immune activation according to the disease subtype. PLoS One. 2012;7(8):e42777.

6 Wardill HR, Gibson RJ, Van Sebille YZ, Secombe KR, Coller JK, White IA, et al. Irinotecan-induced gastrointestinal dysfunction and pain are mediated by common TLR4-dependent mechanisms. Mol Cancer Ther. 2016; 15(6):1376-86

7 Chabot S, Wagner JS, Farrant S, Neutra MR. TLRs regulate the gatekeeping functions of the intestinal follicle-associated epithelium. J Immunol. 2006;176(7):4275-83.

8 Abreu MT. Toll-like receptor signalling in the intestinal epithelium: how bacterial recognition shapes intestinal function. Nat Rev Immunol. 2010;10(2):131-44.
9 Anderson JM, Van Itallie CM, Fanning AS. Setting up a selective barrier at the apical junction complex. Curr Opin Cell Biol. 2004;16(2):140-5.

10 Ma TY, Anderson JM, Turner JR. Tight Junctions and the Intestinal Barrier. In: Physiology of the gastrointestinal tract. Amsterdam: Elsevier; 2012. p. 1043-88.

11 Bein A, Eventov-Friedman S, Arbell D, Schwartz B. Intestinal tight junctions are severely altered in NEC preterm neonates. Pediatr Neonatol. 2018;59(5):464-73.

12 Sodhi CP, Neal MD, Siggers R, Sho S, Ma C, Branca MF, et al. Intestinal epithelial Toll-like receptor 4 regulates goblet cell development and is required for necrotizing enterocolitis in mice. Gastroenterology. 2012;143(3):708-18.e5.

13 Madison BB, Dunbar L, Qiao XT, Braunstein $\mathrm{K}$, Braunstein E, Gumucio DL. Cis elements of the villin gene control expression in restricted domains of the vertical (crypt) and horizontal (duodenum, cecum) axes of the intestine. J Biol Chem. 2002;277(36):33275-83.

14 The Jackson Laboratory. B6(Cg)Tlr4tm1.1Karp/J protocol 26910: standard PCR assay: Tlr4 <tm1.1Karp>. Genotyping protocols database.

15 The Jackson Laboratory. B6.Cg-Tg(Villcre) $997 \mathrm{Gum} / \mathrm{J}$ protocol 24364: standard PCR assay: $\operatorname{Tg}($ Vil-cre $) 997 \mathrm{Gum}$-alternate1. Genotyping protocols database.

16 Wardill HR, Bowen JM, Van Sebille YZ, Secombe KR, Coller JK, Ball IA, et al. TLR4-dependent claudin-1 internalization and secretagogue-mediated chloride secretion regulate irinotecan-induced diarrhea. Mol Cancer Ther. 2016;15(11):2767-79.
17 Howarth GS, Francis GL, Cool JC, Xu X, Byard RW, Read LC. Milk growth factors enriched from cheese whey ameliorate intestinal damage by methotrexate when administered orally to rats. J Nutr. 1996;126:2519-30.

18 Abramoff MD, Magalhaes PJ, Ram SJ. Image processing with ImageJ. Biophotonics Int. 2004;11(7):36-42.

19 Caputi V, Marsilio I, Cerantola S, Roozfarakh M, Lante I, Galuppini F, et al. Toll-like receptor 4 modulates small intestine neuromuscular function through nitrergic and purinergic pathways. Front Pharmacol. 2017;8:350.

20 Forcen R, Latorre E, Pardo J, Alcalde AI, Murillo MD, Grasa L. Toll-like receptors 2 and 4 modulate the contractile response induced by serotonin in mouse ileum: analysis of the serotonin receptors involved. Neurogastroenterol Motil. 2015;27(9):1258-66.

21 Lu P, Sodhi CP, Hackam DJ. Toll-like receptor regulation of intestinal development and inflammation in the pathogenesis of necrotizing enterocolitis. Pathophysiology. 2014; 21(1):81-93

22 Shi YJ, Hu SJ, Zhao QQ, Liu XS, Liu C, Wang $\mathrm{H}$. Toll-like receptor 4 (TLR4) deficiency aggravates dextran sulfate sodium (DSS)-induced intestinal injury by down-regulating IL6, CCL2 and CSF3. Ann Transl Med. 2019; 7(23):713.

23 Dheer R, Santaolalla R, Davies JM, Lang JK, Phillips MC, Pastorini C, et al. Intestinal epithelial toll-like receptor 4 signaling affects epithelial function and colonic microbiota and promotes a risk for transmissible colitis. Infect Immun. 2016;84(3):798-810 
24 Wardill HR, Bowen JM, Al-Dasooqi N, Sultani M, Bateman E, Stansborough R, et al. Irinotecan disrupts tight junction proteins within the gut: implications for chemotherapy-induced gut toxicity. Cancer Biol Ther. 2014; 15(2):236-44.

25 Gourbeyre P, Berri M, Lippi Y, Meurens F, Vincent-Naulleau S, Laffitte J, et al. Pattern recognition receptors in the gut: analysis of their expression along the intestinal tract and the crypt/villus axis. Physiol Rep. 2015;3(2): e12225.

26 Pufnock JS, Cigal M, Rolczynski LS, Andersen-Nissen E, Wolfl M, McElrath MJ, et al. Priming CD8+ T cells with dendritic cells matured using TLR4 and TLR7/8 ligands together enhances generation of CD8+ T cells retaining CD28. Blood. 2011;117(24):6542-51.
27 Salazar F, Awuah D, Negm OH, Shakib F, Ghaemmaghami AM. The role of indoleamine 2,3-dioxygenase-aryl hydrocarbon receptor pathway in the TLR4-induced tolerogenic phenotype in human DCs. Sci Rep. 2017;7:43337.

28 Mukherjee S, Karmakar S, Babu SP. TLR2 and TLR4 mediated host immune responses in major infectious diseases: a review. Braz J Infect Dis. 2016;20(2):193-204.

29 Albrecht V, Hofer TP, Foxwell B, Frankenberger M, Ziegler-Heitbrock L. Tolerance induced via TLR2 and TLR4 in human dendritic cells: role of IRAK-1. BMC Immunol. 2008; 9:69.
30 Frank M, Hennenberg EM, Eyking A, Rünzi $M$, Gerken $G$, Scott P, et al. TLR signaling modulates side effects of anticancer therapy in the small intestine. J Immunol. 2015; 194(4):1983-95.

31 de Groot AM, Thanki K, Gangloff M, Falkenberg E, Zeng X, van Bijnen DCJ, et al. Immunogenicity testing of lipidoids in vitro and in silico: modulating lipidoid-mediated TLR4 activation by nanoparticle design. Mol Ther Nucleic Acids. 2018;11:159-69.

32 Coller JK, Bowen JM, Ball IA, Wardill HR, van Sebille YZ, Stansborough RL, et al. Potential safety concerns of TLR4 antagonism with irinotecan: a preclinical observational report. Cancer Chemother Pharmacol. 2017;79(2): 431-4. 